\title{
Prevalence and pattern of Dyslipidaemia among patients with newly diagnosed type 2 diabetes mellitus in Southern Sri Lanka; a cross sectional study
}

\author{
Herath $\mathbf{H M M}^{1}$, Weerarathna $\mathbf{T P}^{1}$, Weerasinghe $\mathbf{N P}^{2}$ \\ ${ }^{\prime}$ Department of Medicine ${ }^{l}$, Faculty of Medicine, University of Ruhuna, Galle, Sri Lanka. \\ ${ }^{2}$ Department of Microbiology ${ }^{2}$ Faculty of Medicine, University of Ruhuna, Galle, Sri Lanka.
}

Correspondence: Dr. Meththananda Herath

e-mail: herathtp@gmail.com /hmmherath@med.ruh.ac.lk

\begin{abstract}
Introduction: Dyslipidaemia is one of the major risk factors for cardiovascular disease (CVD) in diabetes mellitus. However, the magnitude and characteristic features of dyslipidaemia among Sri Lankans with newly diagnosed diabetes is not yet known. We aimed to study the prevalence and pattern of dyslipidaemia in a cohort of patients with newly diagnosed type 2 diabetes mellitus (T2DM).

Methods: This study was carried out in 403 newly diagnosed T2DM patients attending a specialist diabetic clinic in Southern Sri Lanka. Dyslipidaemia was diagnosed if patients had one or more parameters of lipid profile outside the target values recommended by the American Diabetes Association (ADA).

Results: Diabetic dyslipidaemia was observed in $90 \%$ of patients with females having higher prevalence (94.7\%) than males $(87.0 \%, \mathrm{P}=0.04)$. The most prevalent type of dyslipidaemia was isolated elevation of low density lipoprotein (LDL) cholesterol (84.3\%). Raised triglyceride (TG) and low high density lipoprotein (HDL) were comparatively less common and observed in less than $20 \%$ subjects. Factors such as obesity, waist circumference, age and degree of hyperglycaemia at presentation had no significant effect on the pattern and prevalence of dyslipidaemia.

Conclusions: This study revealed a higher prevalence and a different pattern of dyslipidaemia among newly diagnosed patients with T2DM. Finding of raised LDL with relatively low TG observed in this study was different to the pattern of diabetic Dyslipidaemia described in Caucasians.
\end{abstract}

Keywords: Prevalence and pattern, diabetic dyslipidaemia, newly diagnosed, type 2 diabetes mellitus, Asian ethnicity

\section{Introduction}

Patients with type 2 diabetes mellitus (T2DM) are at higher risk of developing coronary artery disease (CAD) and other vascular diseases such as ischemic stroke. The risk is primarily due to a greater burden of atherogenic risk factors among diabetics, including hypertension, obesity, and dyslipidaemia (1). A number of studies have shown the beneficial effect of treating dyslipidaemia on cardiovascular morbidity and mortality in patients with $\operatorname{T} 2 \mathrm{DM}(2,3)$.
The American Diabetes Association (ADA) recommends screening for dyslipidaemia at the time of diabetes diagnosis and every 5 years thereafter, or more frequently if needed to achieve goals (4). ADA in 2016 update recommends moderate-intensity statin treatment for all patients with diabetes aged $\geq 40$ years and high-dose statins for those with increased cardiovascular risk [e.g., LDL cholesterol $\geq 100 \mathrm{mg} / \mathrm{dL}(2.6 \mathrm{mmol} / \mathrm{L})]$, high blood pressure, smoking, albuminuria, and family history of premature coronary artery disease) (5). 
The typical pattern of dyslipidaemia seen in patients with T2DM is elevated TG and low HDL-C levels, although all lipoproteins have compositional abnormalities (4). Observational studies suggest that low HDL-C is one of the most important risk factor for $\mathrm{CAD}(6,7)$. It is well documented that raised LDL-C and TG are also associated with an increased risk of CAD $(4,8)$. However, differences exist in the morbidity and mortality of diabetes patients between different ethnic groups. Compared to Caucasians, South Asians have a higher risk of developing CAD and other macro-vascular and micro-vascular complications related to diabetes (9). Change in the prevalence and pattern of dyslipidaemia could be a contributing factor for higher risk of CAD among South Asians.

The best time to look at the prevalence and pattern of diabetes dyslipidaemia is at the time of the diagnosis of diabetes as subsequent management with pharmacological agents or non-pharmacological measures can alter both pattern as well as the prevalence of dyslipidaemia. However, according to our knowledge, there were no previous studies on the prevalence and pattern of dyslipidaemia among Sri Lankans with newly diagnosed T2DM. Even for South East Asians, the pattern and the prevalence of dyslipidaemia among newly diagnosed patients with T2DM is not adequately investigated. The knowledge on the pattern and the prevalence of diabetic dyslipidaemia will be useful in the successful management of T2DM particularly in the local setting. Therefore, this study was designed to determine the prevalence and pattern of Dyslipidaemia among newly diagnosed patients with T2DM and evaluate the its associations with risk factors such as hypertension (HT), body mass index (BMI), waist circumference (WC), and fasting blood glucose (FBG).

\section{Methods}

This cross sectional study was conducted in newly diagnosed patients with T2DM presented to a Regional Diabetes Centre, Southern Province of Sri Lanka. This Regional Diabetes Centre attracts patients from whole Southern Province which has a total population of 2.5 million and account for $12 \%$ of Sri Lankan population. The study was carried out from January 2012 to July 2013. Subjects were recruited by convenient sampling method. Posters in public places and collaborations with general practitioners were used to recruit patients with new onset T2DM. Following patients were excluded from the study; patients currently on treatment (pharmacological or non-pharmacological) for dyslipidaemia, patients who have been treated for diabetes in past, those who were unwell due to hyperglycaemia or intercurrent illness, clinically apparent hypothyroidism, nephrotic syndrome and chronic kidney disease, those with extreme body habitus (BMI $<18$ or $>40)$, and when type 1 diabetes or secondary causes for diabetes were more likely on clinical grounds than T2DM. A pretested interviewer-administered questionnaire was used to obtain demographic and medical information such as age, sex, ethnicity, tobacco smoking, and family history of dyslipidaemia, CAD, and metabolic syndrome. Weight, height, and WC were measured and BMI was calculated. All anthropometric measurements were performed by trained nurses adhering to the WHO guidelines, using calibrated equipment. Blood pressure was recorded using an electronic instrument (Omron Corporation, Tokyo, Japan), as the mean of two readings taken five minutes apart.

All chemical analyses were performed in the laboratory attached to the Regional Diabetic Center mentioned above and same method of biochemical analysis was used throughout the study period. Overnight fasting venous blood samples were collected to measure HDL-C and LDL-C, serum TG and glucose. Cholesterol esterase oxidaseperoxidase-amidopyrine method was used to assess serum cholesterol, and for measurement of serum TG glycerol phosphate oxidase-peroxidaseamidopyrine method was used. For HDL cholesterol, direct method poly-ethylene-glycol-pretreated enzymes were used. Approval of the Institutional Ethics Committee of the Faculty of Medicine, University of Ruhuna, was obtained prior to study commencement and written informed consent was obtained from all study subjects in the local language.

All the data were analysed using SPSS 17.0. Dyslipidaemia was diagnosed if any component of lipid profile exceeds recommended targets. Those with dyslipidaemia were further subdivided in to mixed dyslipidaemia (all three parameters outside the recommended ADA targets), combined dyslipidaemia (two parameter outside the 
recommended target) or isolated dyslipidaemia (only one parameter outside the recommended target). Patients with combined dyslipidaemia were further classified in to different patterns of dyslipidaemia (high LDL and TG, high LDL and low HDL, high TG and low HDL). Significance of relationship between following factors with each lipid component level was calculated using Chi square test of independence. Age at presentation $(<45$ or $\geq 45$ years), degree of hyperglycaemia at presentation $\left(\mathrm{HbA}_{1} \mathrm{C}<6.9 \%\right.$ as lower degree of hyperglycaemia and $\mathrm{HbA}_{1} \mathrm{C}$ value $\geq 7.9 \%$ as higher degree of hyperglycaemia), presence of global obesity defined as BMI more than $23 \mathrm{~kg} / \mathrm{m}^{2}$, and presence of central obesity with WC exceeding $80 \mathrm{~cm}$ in females and $90 \mathrm{~cm}$ in males.
T-test and one way ANOVA were used to test significant as appropriate.

\section{Results}

Table 1 shows demographic characteristics of the 403 newly diagnosed patients with T2DM in the study. There were more male $(71.7 \%)$ than female $(28.3 \%)$, and nearly $60 \%$ of the male had younger onset of T2DM (<45 years). Females were significantly older and had lower level of HDL-C than males. Although there was a higher prevalence of central obesity in females $(89 \%)$ compared with males $(59 \%)$, the mean difference was not statistically significant.

Table 1: Baseline characteristics of the study sample according to gender

\begin{tabular}{|c|c|c|c|c|c|c|}
\hline \multirow{2}{*}{\multicolumn{2}{|c|}{$\begin{array}{l}\text { Variables } \\
\text { Number and percentages }\end{array}$}} & \multicolumn{2}{|c|}{ Male $=289$} & \multicolumn{2}{|c|}{ Female $=114$} & \multirow[t]{2}{*}{$p$} \\
\hline & & $\mathbf{n}$ & $\%$ & $\mathbf{n}$ & $\%$ & \\
\hline \multirow[t]{2}{*}{ Age (years) } & & 168 & 58.1 & 42 & 36.8 & - \\
\hline & & 121 & 41.8 & 72 & 63.1 & - \\
\hline \multirow[t]{2}{*}{ BMI } & & 88 & 30.4 & 44 & 38.6 & - \\
\hline & & 201 & 69.5 & 70 & 61.4 & - \\
\hline \multirow{2}{*}{$\begin{array}{l}\text { Waist Circumference } \\
(\mathrm{cm})^{*}\end{array}$} & Normal & 120 & 41.2 & 13 & 11.4 & - \\
\hline & High & 169 & 58.8 & 101 & 88.6 & - \\
\hline \multirow[t]{4}{*}{$\mathrm{HbA}_{1} \mathrm{C}$} & $\leqslant 6.9 \%$ & 77 & 26.6 & 34 & 29.8 & - \\
\hline & $7-7.9 \%$ & 132 & 45.6 & 52 & 45.6 & - \\
\hline & $>7.9 \%$ & 80 & 27.6 & 28 & 24.5 & - \\
\hline & & Mean & SD & Mean & SD & $p$ \\
\hline \multicolumn{2}{|l|}{ Age } & 43.3 & 10.6 & 47.2 & 10.8 & 0.001 \\
\hline \multicolumn{2}{|l|}{ BMI } & 24.5 & 4.9 & 25.0 & 4.8 & 0.36 \\
\hline \multicolumn{2}{|l|}{ Waist Circumference } & 90.3 & 11.1 & 91.4 & 10.4 & 0.34 \\
\hline \multicolumn{2}{|l|}{$\mathrm{HbA} 1 \mathrm{c} \%$} & 6.9 & 1.4 & 7.0 & 1.4 & 0.80 \\
\hline
\end{tabular}

Mean levels of total cholesterol, HDL-C, LDL-C, and TG were 205.3 $\pm 41.8,48.7 \pm 9.3,130.6 \pm 38.3$, and 119.1 \pm 54.8 respectively. Of note, the mean LDL-C of $130.6 \pm 38.3 \mathrm{mg} / \mathrm{dL}$ was well above the LDL-C target of 100 $\mathrm{mg} / \mathrm{dL}$ recommended by ADA. 
Overall, the prevalence of Dyslipidaemia with at least one abnormal lipid parameter was seen in $89 \%$ of patients in the sample with higher prevalence observed in females (94\%) than in males $(87 \%)$. Raised LDL-C was the commonest abnormality affecting 84\%; however, raised TG and low HDL-C were comparatively less common accounting for $19 \%$ and $18 \%$ respectively. Females had higher prevalence of unfavorable lipid abnormalities including raised LDL-C $(91 \%)$ and low HDL$\mathrm{C}(38 \%)$. However, raised TG was more prevalent in males than in females (Figure 1).
Table 2 presents the pattern of dyslipidaemia in newly diagnosed patients with T2DM according to their gender. Among males, isolated raised LDL-C was the commonest pattern seen around $60 \%$ and combined dyslipidaemia with raised LDL-C and TG was observedaround $15 \%$. Mixed dyslipidaemia with all three abnormal lipid parameters (TG, LDL-C and low HDL-C) was rare (1.4\%) in males. In females too, isolated raised LDL-C was the most common pattern of dyslipidaemia (46.5\%) followed by combined dyslipidaemia (32\%) with raised LDL-C and low HDL-C. However, combination of raised LDL-C and TG was less common in females $(7.9 \%)$ in compared to males $(15.6 \%)$.

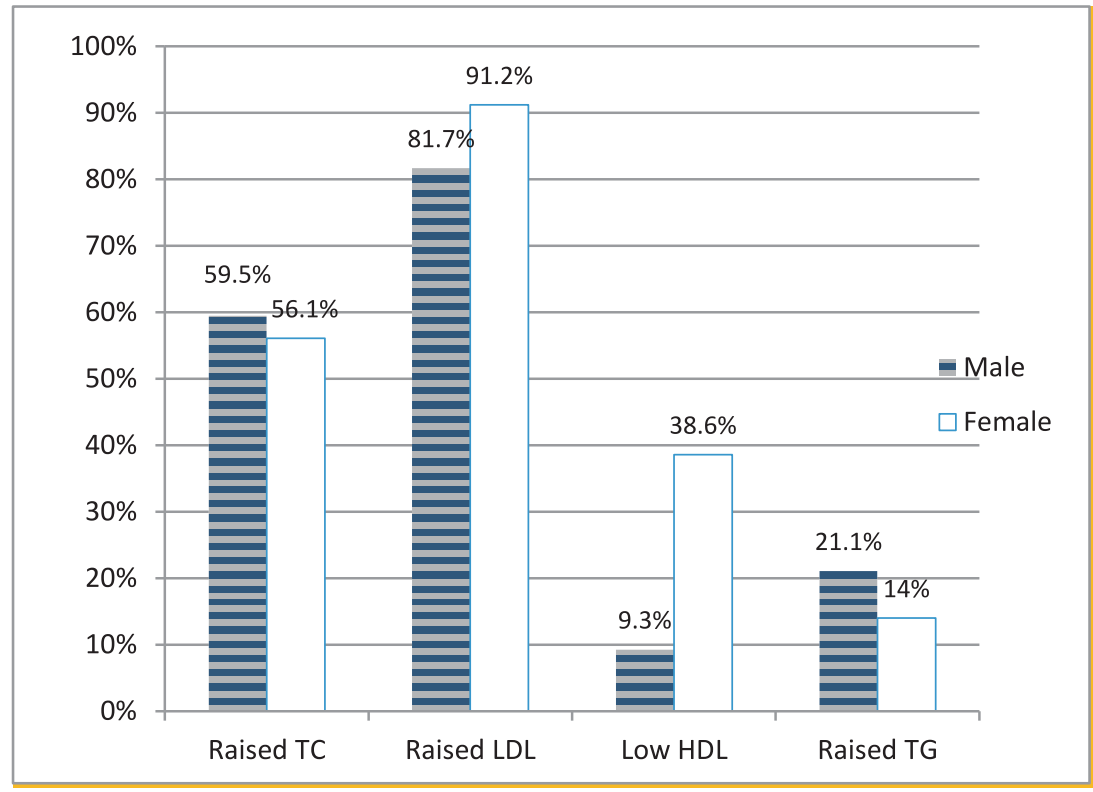

Figure 1: Lipid abnormalities according to gender

Table 2: Pattern of Dyslipidaemia according to gender

\begin{tabular}{llllc}
\hline & \multicolumn{2}{c}{ Males(289) } & \multicolumn{2}{c}{ Females(114) } \\
\hline Pattern of dyslipidaemia & $\mathrm{n}$ & $\%$ & $\mathrm{n}$ & $\%$ \\
Isolated single parameter dyslipidaemia & 184 & 63.7 & 57 & 50.1 \\
$\quad$ Raised LDL-C & 170 & 58.8 & 53 & 46.5 \\
$\quad$ Raised TG-C & 10 & 3.5 & 2 & 1.8 \\
$\quad$ Low HDL-C & 4 & 1.4 & 2 & 1.8 \\
Combined dyslipidaemia & 64 & 22.2 & 46 & 40.4 \\
$\quad$ High TG and low HDL-C & 2 & 0.7 & 0 & 0 \\
$\quad$ HIGH TG and high LDL-C & 45 & 15.6 & 9 & 7.9 \\
$\quad$ High LDL and low HDL-C & 17 & 5.9 & 37 & 32.5 \\
Mixed dyslipidaemia & 4 & 1.4 & 5 & 4.4 \\
(high TG, high LDL-C and low HDL-C) & & & & \\
Total & 252 & 87.2 & 108 & 94.7 \\
\hline
\end{tabular}


We analysed prevalence and pattern of dyslipidaemia according to degree of hyperglycemia at the time of diagnosis of T2DM. $\mathrm{HbA}_{1} \mathrm{C}$ value less than or equal to $6.9 \%$ was considered as lower degree of hyperglycaemia whereas values $7.9 \%$ or more was considered as higher degree of hyperglycaemia. As shown in Table 3 there was no significant effect of degree of hyperglycaemia on the pattern and the prevalence of dyslipidaemia. However, lower degree of hyperglycaemia was more commonly associated with isolated Dyslipidaemia even though it did not reach statistical significance.
We also evaluated whether the age at diagnosis of T2DM had any effect on the prevalence and pattern of dyslipidaemia. As shown in Table 4, raised TG was significantly different between groups with higher mean value observed in patients with younger (25-35 years) onset T2DM compared older onset. Even though not reaching significant level, other lipid parameters too (raised LDL, and low HDL) were higher in younger individuals.

Table 3: Lipid abnormalities according to degree of hyperglycaemia

\begin{tabular}{lccc}
\hline \multirow{2}{*}{ Prevalence of dyslipidaemia } & \multicolumn{3}{c}{ Degree of hyperglycaemia } \\
\cline { 2 - 4 } & $\mathrm{HbA}_{1} \mathrm{C} \$ 6.9 \%$ & $\mathrm{HbA}_{1} \mathrm{C} \geqslant 7.9 \%$ & $p$ \\
& $(\%)$ & $(\%)$ & 0.83 \\
\hline Raised LDL & 86.4 & 87.0 & 0.11 \\
Raised TG & 10.8 & 19.4 & 0.24 \\
Low HDL & 13.5 & 20.3 & 0.48 \\
Total & 88.2 & 90.7 & $p$ \\
\hline Pattern of dyslipidaemia & $\%$ & $\%$ & 0.12 \\
\hline Mixed dyslipidaemia & 0 & 2.7 & 0.27 \\
Combined dyslipidaemia & 21.6 & 29.6 & 0.09 \\
Isolated dyslipidaemia & 67.5 & 59.2 & 0.34 \\
No Dyslipidaemia & 10.8 & 09 & \\
\hline
\end{tabular}

TC - total cholesterol, LDL - low density lipoprotein, HDL - low high density lipoprotein, TG -triglycerides

Table 4: Lipid abnormalities according to age at diagnosis

\begin{tabular}{|c|c|c|c|c|c|c|c|c|c|c|c|c|c|}
\hline \multirow{2}{*}{$\begin{array}{c}\text { Age } \\
\text { categories }\end{array}$} & \multirow[b]{2}{*}{$\mathbf{n}$} & \multicolumn{3}{|c|}{ TC } & \multicolumn{3}{|c|}{ LDL } & \multicolumn{3}{|c|}{ TG } & \multicolumn{3}{|c|}{ HDL } \\
\hline & & Mean & SD & $p^{*}$ & mean & SD & $p^{*}$ & mean & SD & $p^{* *}$ & mean & SD & $p^{*}$ \\
\hline $15-24$ & 12 & 209.3 & 23.4 & \multirow[t]{5}{*}{0.19} & 130.0 & 33.4 & \multirow[t]{5}{*}{0.13} & 125.0 & 58.4 & \multirow[t]{5}{*}{0.01} & 48.3 & 6.6 & \multirow[t]{5}{*}{0.42} \\
\hline $25-34$ & 64 & 216.7 & 43.2 & & 139.6 & 42.3 & & 140.3 & 69.3 & & 46.4 & 9.8 & \\
\hline $35-44$ & 140 & 207.4 & 47.5 & & 131.4 & 42.9 & & 124.4 & 63.8 & & 47.7 & 9.2 & \\
\hline $45-54$ & 131 & 194.0 & 52.3 & & 124.0 & 39.3 & & 108.1 & 46.8 & & 47.7 & 12.9 & \\
\hline $55-64$ & 60 & 198.5 & 44.1 & & 125.4 & 41.0 & & 105.1 & 30.4 & & 50.1 & 10.0 & \\
\hline
\end{tabular}

TC - total cholesterol, LDL - low density lipoprotein, HDL - low high density lipoprotein, TG - triglycerides.

*According one way ANOVA. ** Post Hoc test revealed that there was significant difference exist between age category of 25-34 with 45-54 $(\mathrm{p}<0.01)$ and with 55-64 $(\mathrm{p}=0.01)$. 
We examined the prevalence of dyslipidaemia according to BMI and waist circumference. Raised LDL was the commonest abnormality in all BMI categories followed by raised TG. Overall BMI had no effect on the prevalence of dyslipidaemia. Central obesity measured according to waist circumference had no significant effect on the overall prevalence of dyslipidaemia.

\section{Discussion}

In the wake of rising incidence of diabetes and cardiovascular diseases in Sri Lanka, this study is a step towards evaluating the prevalence and the pattern of diabetic dyslipidaemia. Furthermore, this is the first paper on diabetes dyslipidaemia in Sri Lankans with newly diagnosed type 2 diabetes mellitus. This study revealed close to $90 \%$ patients had some form of dyslipidaemia at the time of diagnosis of T2DM. Transferred on to the total diabetes population in Sri Lanka, this figure may imply that approximately 1.8 million Sri Lankans with diabetes may be affected by dyslipidaemia.

The mean levels of total cholesterol and LDL-C found in our study were comparable to the mean total cholesterol levels and LDL-C reported in the United Kingdom Prospective Diabetes Study (UKPDS) $(10,11)$. However, raised TG observed in our study $(113 \mathrm{mg} / \mathrm{dL}$ in males and $121 \mathrm{mg} / \mathrm{dL}$ in females) was much lower than the mean TG levels of UKPDS $(159 \mathrm{mg} / \mathrm{dL}$ in both genders) (11). Mean HDL-C level of $51.5 \mathrm{mg} / \mathrm{dL}$ in males and $48.6 \mathrm{mg} / \mathrm{dL}$ in females found in our study were also different to the mean HDL-C levels of $39 \mathrm{mg} / \mathrm{dl}$ in males and $43 \mathrm{mg} / \mathrm{dl}$ in females reported in UKPDS study. The UKPDS study also reported higher prevalence of adverse Dyslipidaemia in females than in males (11). Such difference was not observed in our study; however there was a significantly lower HDL-C level observed in females in our study.

The most prevalent type of dyslipidaemia in this study was raised LDL followed by hypertriglyceridemia. Prevalence of low HDL was comparatively low and seen in just $18 \%$ of the sample. These findings are different to diabetic dyslipidaemia described in Caucasians $(4,11,13)$. However, some studies including one conducted in Sri Lanka also have reported higher LDL cholesterol level than TG in patients with T2DM (14). Raised LDL cholesterol with relatively lower TG was also reported among patients with diabetes in other developing countries such as Nigeria, and India $(15,17)$. But, unlike in our study most of these studies showed higher prevalence of low HDL.

There could be many reasons for the different pattern of diabetic dyslipidaemia in the cohort of patients that we studied. Unlike some of the published studies on diabetic dyslipidaemia, we studied only the newly diagnosed patients with T2DM who were not on any treatment for dyslipidaemia. Therefore, the pattern of Dyslipidaemia observed in this study reflects the true picture of diabetes dyslipidaemia. Higher prevalence of TG with low LDL reported in previous studies could be an effect of statins as it lowers LDL more than TG. The typical changes of raised TG with low HDL-C seen in diabetic dyslipidaemia is thought to be due to insulin resistance together with dysfunction of the enzyme lipoprotein lipase (LPL). It was postulated that insulin resistance in adipocytes promote lipolysis, resulting in excessive free fatty acid (FFA) release into the blood and higher production of TG-rich very low-density lipoproteins (VLDL) by liver. Higher production of VLDL together with blunted LPL activity contributes raised TG levels (18). Therefore, one of the possibilities for comparatively lower levels of TG seen in our study is either lower degree of insulin resistance or higher LPL activity or both. Further studies are needed to evaluate insulin resistance among patients with raised LDL-C and raised TG to answer this question. Higher prevalence of raised LDL in this study may indicate higher prevalence of raised LDL in the general population. Even though the prevalence of lipid abnormalities in the general population of Sri Lanka is not well studied, studies conducted in urban India had shown higher prevalence of raised LDL in general population $(19,20)$. One of the other possibilities is the rising incidence of obesity in the local population (21). As substantial proportion of subjects $(60 \%)$ were obese in our study, it could contribute for raised LDL levels.

Gender differences in the pattern of altered plasma lipids observed in this study are similar to major epidemiological studies from Western populations. However, in contrast to females who were more 
dyslipidemic with higher LDL and triglycerides in those studies, our study revealed higher proportions of males (21\%) having raised triglycerides compared to females $(14 \%)$. Our study also showed that diabetes occurring in young individuals ( $<45$ years) had significantly higher prevalence of elevated triglycerides. Even though not reaching significant level, other lipid parameters too (raised LDL, and low HDL) were higher in younger individuals $(<45$ years). This may be significant in the light of the finding that new onset diabetes in the South Asians is occurring at a relatively younger age and they need to be treated accordingly with appropriate measures (22).

Other factors such as degree of hyperglycaemia at diagnosis, BMI, and WC showed no significant effect on prevalence and pattern of dyslipidaemia. Therefore, predicting underlying Dyslipidaemia based on BMI, and WC may not be appropriate.

\section{Conclusions}

The prevalence of dyslipidaemia is high among newly diagnosed patients with T2DM affecting over $90 \%$. In both genders, the most common pattern of dyslipidaemia is isolated raised LDL cholesterol followed by combined dyslipidaemia with raised LDL and raised TG in males and combined dyslipidaemia with raised LDL and low HDL in females. Typical pattern of diabetic dyslipidaemia with raised TG and low HDL is not observed in the majority. Other associated and contributing factors of dyslipidaemia such as obesity, waist circumference, and degree of hyperglycaemia at diagnosis had no significant effect on the pattern and prevalence of dyslipidaemia. Based on the findings of our study, we recommend lipid profile to be carried out in all patients at the diagnosis of T2DM and decision to perform lipid profile should not be based on factors such as obesity and degree of hyperglycaemia.

\section{Limitations}

The main limitations of our study include its single center study design. Therefore, generalisation of our findings accurately to whole diabetes population of Sri Lanka may not be plausible. The other limitation is not performing lipoproteins and genetic studies for further characterization of dyslipidaemia.

\section{Acknowledgements}

We would like to thank the participants of this study, all the staff of the Diabetes Center, Galle and Consultants who kindly consented to use their patients. A special word of thank is extended to K. M Kumuduni de Silva and K.S.M. Weerarathna for laboratory assistance, and Dr. S.P. Mohotti, Dr. C.M. De Silva and Dr. L. Fonseka for their assistance in conducting this study.

Disclosure: We would like to declare that we have no conflicts of interest in this work.

\section{References}

1. Turner RC, Millns H, Neil HA, Stratton IM, Manley SE, Matthews DR, et al. Risk factors for coronary artery disease in non-insulin dependent diabetes mellitus: United Kingdom Prospective Diabetes Study (UKPDS: 23). BMJ 1998; 316(7134): 823-8.

2. Cholesterol Treatment Trialists C, Mihaylova B, Emberson J, Blackwell L, Keech A, Simes J, et al. The effects of lowering LDL cholesterol with statin therapy in people at low risk of vascular disease: meta-analysis of individual data from 27 randomised trials. Lancet 2012; 380(9841): 581-90.

3. Battaggia A, Font M. Statins for people at low risk of cardiovascular disease. Lancet. 2012;380(9856): 1815.

4. Haffner SM, American Diabetes A. Dyslipidaemia management in adults with diabetes. Diabetes Care. 2004; 27(Suppl 1): S68-71.

5. Cardiovascular Disease and Risk Management. Diabetes care 2016;39(Suppl 1):S60-71.

6. Miller M, Kwiterovich PO. Isolated low HDL-cholesterol as an important risk factor for coronary heart disease. European Heart Journal 1990; 11(Suppl H): 9-14.

7. Haffner SM. Management of Dyslipidaemia in adults with diabetes. Diabetes care 1998;21(1):160-78.

8. American Diabetes A. Management of Dyslipidaemia in adults with diabetes. Diabetes care 2000; 23(Suppl 1): S57-60.

9. Karter AJ, Ferrara A, Liu JY, Moffet HH, Ackerson LM, Selby JV. Ethnic disparities in diabetic complications in an insured population. JAMA: the journal of the American Medical Association. 2002; 287(19): 2519-27.

10. Davis TM, Millns H, Stratton IM, Holman RR, Turner RC. Risk factors for stroke in type 2 diabetes mellitus: United Kingdom Prospective Diabetes Study (UKPDS) 29. Archives of Internal Medicine 1999; 159(10): 1097-103. 
11. UK Prospective Diabetes Study 27. Plasma lipids and lipoproteins at diagnosis of NIDDM by age and sex. Diabetes care 1997; 20(11): 1683-7.

12. Haffner SM, Goldberg RB. New strategies for the treatment of diabetic dyslipidaemia. Diabetes care 2002; 25(7): 1237-9.

13. Stern MP, Haffner SM. Dyslipidaemia in type II diabetes. Implications for therapeutic intervention. Diabetes care 1991; 14(12): 1144-59.

14. Hettihewa LMG, Jayasinghe SS, et al. Lipid abnormalities in type 2 diabetes mellitus patients in Srilanka. Galle Medical Journal 2007.

15. Subburam R, Manohar CR, Subramaniyam P, Sachithanantham S, Paul AV, Sankarapandian M. Dyslipidaemia among type 2 diabetes mellitus patients in a rural hospital in Erode district, Tamilnadu. Journal of the Indian Medical Association 2013; 111(1): 10-3.

16. Ogbera AO, Fasanmade OA, Chinenye S, Akinlade A. Characterization of lipid parameters in diabetes mellitus a Nigerian report. International Archives of Medicine 2009; 2(1): 19.

17. Jisieike-Onuigbo NN, Unuigbe EI, Oguejiofor CO. Dyslipidaemias in type 2 diabetes mellitus patients in Nnewi South-East Nigeria. Annals of African Medicine 2011; 10(4): 285-9.
18. Ginsberg HN. Diabetic dyslipidaemia: basic mechanisms underlying the common hypertriglyceridaemia and low HDL cholesterol levels. Diabetes 1996; 45(Suppl 3): S27-30.

19. Dammerman M, Breslow JL. Genetic basis of lipoprotein disorders. Circulation 1995;91(2): 505-12.

20. Misra A, Shrivastava U. Obesity and Dyslipidaemia in South Asians. Nutrients 2013;5(7): 2708-33.

21. Katulanda P, Jayawardena MA, Sheriff MH, Constantine GR, Matthews DR. Prevalence of overweight and obesity in Sri Lankan adults. Obesity reviews: an official Journal of the International Association for the Study of Obesity 2010; 11(11): 751-6.

22. Mohan V, Deepa M, Deepa R, Shanthirani CS, Farooq S, Ganesan A, et al. Secular trends in the prevalence of diabetes and impaired glucose tolerance in urban South India - the Chennai Urban Rural Epidemiology Study (CURES-17). Diabetologia 2006; 49(6): 1175-8. 